\title{
Optical pumping of a single hole spin in a $p$-doped quantum dot coupled to a metallic nanoparticle
}

\author{
M. A. Antón, F. Carreño, Sonia Melle, Oscar G. Calderón, and E. Cabrera-Granado \\ Facultad de Óptica y Optometría, Universidad Complutense de Madrid, Calle de Arcos de Jalón 118, 28037 Madrid, Spain
}

Mahi R. Singh

Department of Physics and Astronomy, The University of Western Ontario, London, Ontario N6A 3K7, Canada

(Received 21 December 2012; revised manuscript received 19 April 2013; published 7 May 2013)

\begin{abstract}
The preparation of quantum states with a defined spin is analyzed in a hybrid system consisting of a $p$-doped semiconductor quantum dot (QD) coupled to a metallic nanoparticle. The quantum dot is described as a four-level atom-like system using the density matrix formalism. The lower levels are Zeeman-split hole spin states and the upper levels correspond to positively charged excitons containing a spin-up, spin-down hole pair and a spin electron. A metallic nanoparticle with spheroidal geometry is placed in close proximity to the quantum dot, and its effects are considered in the quasistatic approximation. A linearly polarized laser field drives two of the optical transitions of the QD and produces localized surface plasmons in the nanoparticle which act back upon the QD. The frequencies of these localized plasmons are very different along the two principal axes of the nanoparticle, thus producing an anisotropic modification of the spontaneous emission rates of the allowed optical transitions which is accompanied by local-field corrections. This effect translates into a preferential acceleration of some of the optical pathways and therefore into a fast initialization of the QD by excitation with a short optical pulse. The population transfer between the lower levels of the QD and the fidelity is analyzed as a function of the nanoparticle's aspect ratio, the external magnetic field, and the Rabi frequency of the driving field. It is also shown that the main effect of the local-field corrections is a lengthening of the time elapsed to reach the steady-state. The hole spin is predicted to be successfully cooled from 5 to $0.04 \mathrm{~K}$ at a magnetic field of $4.6 \mathrm{~T}$ applied in the Voigt geometry.
\end{abstract}

DOI: 10.1103/PhysRevB.87.195303

PACS number(s): 78.67.Qa

\section{INTRODUCTION}

Semiconductor quantum-dots (QDs) are currently under consideration as candidates to store quantum bits (qubits) for the future implementation of quantum information processing devices, in view of their scalability, feasibility of coherent manipulations, and strong robustness against relaxation. ${ }^{1,2}$ The resonant excitation of single QDs has enabled the observation of Rabi oscillations, ${ }^{3}$ and coherent manipulation of excitons. ${ }^{4}$ These advances, in turn, have strengthened various proposals, including those regarding optical accessing of spins in QDs. ${ }^{5}$

A key element for quantum information processing is the initial quantum state preparation, which requires qubits initialized in a known state for computation and gate operations. The electron spin has been considered as a potential qubit because its strong quantum confinement suppresses the spin-orbit interaction. However, due to the strong interaction with the reservoir of QD nuclei spin, the time-averaged coherence of an electron spin is limited to time intervals in the order of $10 \mathrm{~ns}^{6}$ The fast electron spin relaxation and decoherence can be suppressed in several ways. Two examples, including spin-echo techniques ${ }^{7}$ as well as polarizing the nuclei spin, ${ }^{8-10}$ have achieved electron-spin coherence times on the microsecond scale. ${ }^{7,11}$ The initialization of an electron spin state (optical pumping or laser cooling of an electron spin) has been demonstrated in a singly charged QD by applying a magnetic field in the Faraday geometry. ${ }^{12}$ In this scheme the initialization rate is predicted to be around $3 \times 10^{5} \mathrm{~s}^{-1}$ with near-unity fidelity.

A different approach to minimize the interaction with the reservoir of QD nuclei spin relies on confining a single hole spin to a QD. The $p$-type atomistic Bloch function of a hole wave function has a node at the position of the QD nuclei, thus leading to a significant reduction in the hyperfine contact coupling Hamiltonian. ${ }^{13}$ Using this approach, Brunner et al. ${ }^{14}$ have experimentally obtained a hole spin relaxation time on the order of $1 \mathrm{~ms}$.

Another important requirement for quantum computation is a continuous supply of ancillary qubits for quantum error correction. This demands that the state initialization speed must be much faster than the quantum state decoherence rate. Theoretical studies have shown that the Voigt geometry allows initialization times shorter than the typical spin coherence time but with a reduction of the maximum attainable fidelity. ${ }^{15}$ The spin cooling rate has been measured to be in the order of $10^{9} \mathrm{~s}^{-1}$ (see Ref. 16), which is a great improvement over previous results. Loo et al. ${ }^{17}$ have predicted that a further increase in the state initialization rate can be achieved by coupling the QD with a high quality factor optical cavity. This theoretical proposal relies on the strong modification of the local density of states in a cavity system. The initial spin state is optically depleted using a light pulse of controlled area followed a preferential, Purcell-accelerated deexcitation toward the desired final state, or target state.

In this work we propose a scheme to obtain a fast hole spin initialization with high fidelity in a hybrid system consisting of a $p$-doped QD and a metallic nanoparticle (MNP) by proper adjustment of the shape of the MNP, the MNP-to-QD distance, and the Rabi frequency of the driving field. The underlying physical mechanism relies on the fact that metallic nanoparticles with sizes smaller than the wavelength of light in vacuum exhibit strong dipolar excitations in the form of localized surface plasmon resonances, which are nonpropagating 
excitations of the conduction electrons in the MNP coupled to the light field. ${ }^{18}$ These localized surface plasmons are tightly confined spatially, leading to a large resonant enhancement of the local field inside and near the MNP. ${ }^{19-22}$ In addition, the local density of states is dramatically altered by the MNP, which results in a modification of the spontaneous emission rates of the QD's optical transitions. ${ }^{22-26}$ Thus, the coupling of a single MNP to a QD can be exploited as a nanoscale cavity ${ }^{27}$ offering a route to size reduction. We theoretically explore how this localized plasmon-exciton interaction can lead to the quantum control of a single hole spin in a positively charged $p$-doped semiconductor quantum dot (QD). In particular, we investigate how the anisotropic enhancement of the QD decay rates due to a spheroidal geometry of the MNP can modify the time scale and the efficiency of spin state initialization. We will consider the so-called Voigt configuration in which the magnetic field is applied along the growth plane of the QD. We present numerical simulations for a representative case of QD, with values of the oscillator strengths, decay rates, and the characteristic energy levels taken from experimental studies. ${ }^{14}$

The paper is organized as follows: Section II establishes the model, i.e., the Hamiltonian of the system and the time-evolution equations of the atomic operators assuming the rotating wave approximation. Section III deals with the numerical simulations and explores the possibility of obtaining fast spin initialization via plasmonic interaction. Finally, Sec. IV summarizes the main conclusions.

\section{THEORETICAL MODEL}

We consider an InAs/GaAs self-assembled QD with growth direction along the $Z$ axis. Self-assembly of QDs is based on growing layers of semiconductors with different lattice

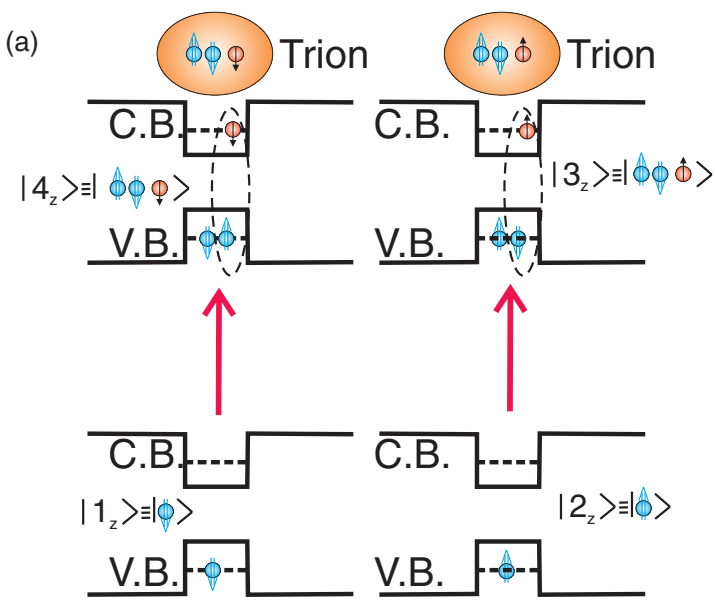

constants on top of each other via the Stranski-Krastanov method. The lattice mismatch causes strain between both layers which results in additional strain energy. After reaching a critical thickness this strain energy is minimized by reducing the contact area between both layers. As a result the top layer forms little islands. In the case of a $p$-doped back contact the QDs are separated by a layer several nanometers thick from a Fermi sea of holes. An applied bias between the top gate and the back contact controls the QD charge state (see Refs. 28 and 29 for specific details regarding experimental examples of such a device).

Let us consider a QD which is charged with a single hole. The ground hole states are labeled $\left|1_{z}\right\rangle \equiv|\Downarrow\rangle$ and $\left|2_{z}\right\rangle \equiv|\Uparrow\rangle$, and the excited trion states $\left(X^{1+}\right)$ are $\left|3_{z}\right\rangle \equiv|\Downarrow \Uparrow \uparrow\rangle$ and $\left|4_{z}\right\rangle \equiv|\Downarrow \Uparrow \downarrow\rangle$. Here $\Uparrow(\Downarrow)$ and $\uparrow(\downarrow)$ denote a heavy hole (HH) and an electron with spins along (against) the $Z$ axis. Hole and electron spin states are naturally degenerate at zero magnetic field. Spin eigenvalues are $\pm 1 / 2$ for electrons and $\pm 3 / 2$ for holes. The energy level diagram of the QD is shown in Fig. 1(a). Angular momentum conservation restricts the optically active transition to excitations where the difference in spin between initial and final states is one. Hence, the $\left|1_{z}\right\rangle \leftrightarrow$ $\left|4_{z}\right\rangle$ transition can only be driven by a $\sigma^{+}$polarized laser field, the transition $\left|2_{z}\right\rangle \leftrightarrow\left|3_{z}\right\rangle$ is restricted to $\sigma^{-}$polarization, and the transitions $\left|2_{z}\right\rangle \leftrightarrow\left|4_{z}\right\rangle$ and $\left|1_{z}\right\rangle \leftrightarrow\left|3_{z}\right\rangle$ remain dark. In order to implement a fast spin state initialization the dark transitions must become bright. This can be realized by applying an external magnetic field along the $X$ axis, perpendicular to the sample growth direction ( $Z$ axis), in the so-called Voigt geometry. The magnetic field introduces an energy shift depending on the carrier spin direction as

$$
E_{\text {Zeeman }}^{h(e)}=\frac{1}{2} \mu_{B}\left(g^{h(e)}\right) B_{x}
$$

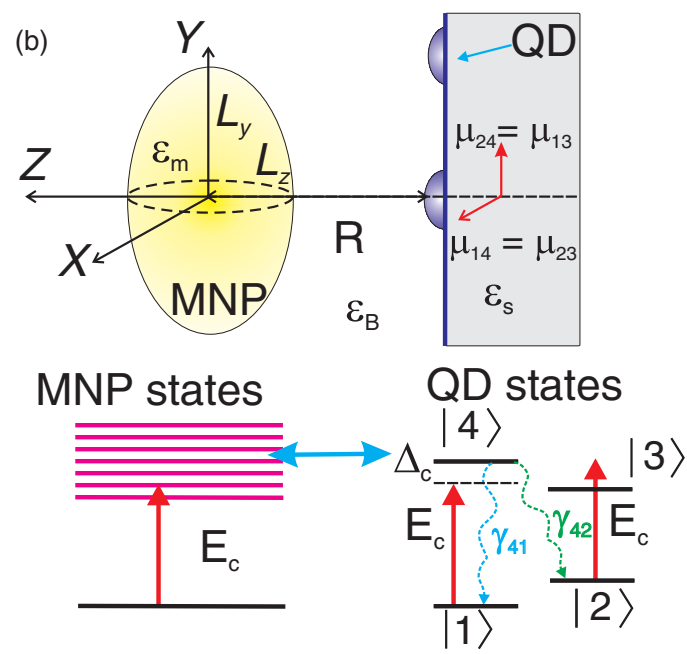

FIG. 1. (Color online) (a) Four-level scheme illustrating the ground and excited states of self-assembled QDs. C.B. and V.B. stand for the conduction and valence bands. (b) Metallic nanospheroid placed close to the QDs. The MNP has a semiminor axis $L_{z}=L_{x}$, a semimajor axis $L_{y}$, and a dielectric constant $\epsilon_{m}$. The dielectric constants of the QD and the host medium are $\epsilon_{s}$ and $\epsilon_{B}$, respectively. Atomic states of the positively charged QD and the MNP. The QD atomic states in the $X$ basis are $|1\rangle$ and $|2\rangle$ for the hole spin eigenvectors, split by the Zeeman effect; the upper levels $|3\rangle$ and $|4\rangle$ are $X^{1+}$ excitons consisting of two spin-paired holes in the V.B. and an unpaired electron with spin $\pm 1 / 2$ in the C.B. The straight lines indicate the driving field linearly polarized along the $X$ axis. The wavy lines indicate the spontaneous relaxation from the upper level $|4\rangle$ to the ground states. In addition, $\gamma_{32}$ and $\gamma_{31}$ are the decay rates of the upper level $|3\rangle$ to the ground states transitions (not shown). 
Here, $E_{\text {Zeeman }}^{h(e)}$ is the Zeeman energy shift relative to $B_{x}=0 \mathrm{~T}$, $B_{x}$ being the magnetic field, and $\mu_{B}$ stands for the Bohr magneton. The quantity $g^{h(e)}$ is the Landé factor of carrier $h(e)$. This magnetic field causes a reference frame transformation from the $Z$ to $X$ basis. The ground hole states are $|1\rangle \equiv$ $\left|\Downarrow_{x}\right\rangle=\frac{1}{\sqrt{2}}(|\Downarrow\rangle-|\Uparrow\rangle)$ and $|2\rangle \equiv\left|\uparrow_{x}\right\rangle=\frac{1}{\sqrt{2}}(|\Downarrow\rangle+|\Uparrow\rangle)$. In addition, the electron spin states are $\left|\uparrow_{x}\right\rangle=\frac{1}{\sqrt{2}}(|\uparrow\rangle+|\downarrow\rangle)$ and $\left|\downarrow_{x}\right\rangle=\frac{1}{\sqrt{2}}(|\uparrow\rangle-|\downarrow\rangle)$, while the mixed trion states are $|4\rangle=\left|\uparrow_{x} \Downarrow_{x} \downarrow_{x}\right\rangle$ and $|3\rangle=\left|\uparrow_{x} \Downarrow_{x} \uparrow_{x}\right\rangle$. Applying the $Z$-basis optical selection rules $\left(\left\langle 1_{z}\left|\sigma^{+}\right| 4_{z}\right\rangle \neq 0\right.$ and $\left.\left\langle 2_{z}\left|\sigma^{-}\right| 3_{z}\right\rangle \neq 0\right)$ leads to new optical selection rules which now read

$$
\begin{array}{ll}
\left\langle 4\left|E_{x}\right| 1\right\rangle \neq 0, & \left\langle 4\left|E_{y}\right| 1\right\rangle=0, \\
\left\langle 3\left|E_{x}\right| 2\right\rangle \neq 0, & \left\langle 3\left|E_{y}\right| 2\right\rangle=0, \\
\left\langle 4\left|E_{x}\right| 2\right\rangle=0, & \left\langle 4\left|E_{y}\right| 2\right\rangle \neq 0, \\
\left\langle 3\left|E_{x}\right| 1\right\rangle=0, & \left\langle 3\left|E_{y}\right| 1\right\rangle \neq 0 .
\end{array}
$$

Here,

$$
E_{x}=\frac{1}{2}\left(\sigma^{+}+\sigma^{-}\right), \quad E_{y}=\frac{1}{2}\left(\sigma^{+}-\sigma^{-}\right)
$$

was used. Now each hole spin ground state can be linked to two exciton states via linearly and orthogonally polarized transitions. The four levels of the system in the Voigt configuration are depicted in Fig. 1(b). The analysis of the experiments carried out by Brunner et al. ${ }^{14,28,29}$ confirms that this level diagram provides an accurate description of the current system. Similar arrangements have been previously considered in other works. ${ }^{16,30-32}$

Our scheme to initialize a single hole spin is presented in Fig. 1(b): a linearly polarized laser $E_{c}$ along the $X$ axis drives the hole state $|1\rangle$ to an exciton state $|4\rangle$. The system can then relax into the desired state $|2\rangle$ or return to state $|1\rangle$, where it can be excited once again. Note that the same laser also drives the $|2\rangle \leftrightarrow|3\rangle$ transition, although in a nonresonant way.

In order to shorten the characteristic time to transfer the population to the target state $|2\rangle$, we pursue to selectively accelerate the decay rate from the trion state $|4\rangle$ to the target state $|2\rangle$, while keeping the other decay rate transition $|4\rangle \leftrightarrow|1\rangle$ nearly unchanged. To accomplish this a MNP with nanospheroid geometry is placed close to the QD as shown in Fig. 1(b). The center-to-center distance between the QD and the MNP is denoted as $R$. The semiminor axis and semimajor axis of the MNP are $L_{x}=L_{z}$ and $L_{y}$, respectively [Fig. 1(b)]. We define the aspect ratio of the nanospheroid as $q=L_{y} / L_{z}$. We will show that the appropriate engineering of the shape of the MNP will result in a way to tailor the dynamics of the QD-MNP hybrid system. The driving field $E_{c}$ is assumed to be spatially uniform over the relatively small dimensions of the hybrid system, and this allows us the use of the quasistatic approximation $^{33}$ when describing the interaction of the light field with the MNP. The dielectric function of the QD is $\epsilon_{s}$, while that of the host medium is denoted as $\epsilon_{B}$ [see Fig. 1(b)]. The dielectric function of the MNP, $\epsilon_{m}(\omega)$, is taken in a renormalized Drude approximation as

$$
\epsilon_{m}(\omega)=\epsilon_{\infty}-\frac{\omega_{p}^{2}}{\omega^{2}+i \gamma_{p} \omega},
$$

where $\epsilon_{\infty}$ is the high-frequency limit of the metal dielectric function, $\omega_{p}$ is the bulk plasma frequency, and $\gamma_{p}$ is the Landau damping constant.

The system is driven by a classical light field with amplitude $E_{c}$ and angular frequency $\omega_{L}$, which is assumed to be linearly polarized in the $X$ direction and is given by

$$
\overrightarrow{\mathcal{E}}_{c}=\frac{1}{2} \hat{u}_{x} E_{c}(t) e^{-i \omega_{L} t}+\text { c.c. },
$$

$\hat{u}_{x}$ being the unitary vector along the $X$ axis. Therefore, the driving field only couples transitions $|1\rangle \leftrightarrow|4\rangle$ and $|2\rangle \leftrightarrow|3\rangle$.

The time evolution of the density matrix reads

$$
\frac{\partial \rho}{\partial t}=-\frac{i}{\hbar}[H, \rho]-\mathcal{L} \rho,
$$

where $H$ is the Hamiltonian of the hybrid system and is explicitly given by

$$
H=\hbar \sum_{j=1}^{4} \omega_{j} \sigma_{j j}-\frac{1}{2}\left[\mu_{41} \sigma_{41}+\mu_{32} \sigma_{32}\right] E_{\mathrm{QD}}^{(x)} e^{-i \omega_{L} t}+\text { H.c. },
$$

In the above expression, $\sigma_{i j}=|i\rangle\langle j|$ are the excitonic operators and $\mu_{i j}$ is the dipole moment of the transition $|i\rangle \leftrightarrow|j\rangle$. The quantity $E_{\mathrm{OD}}^{(x)}$ represents the slowly-varying amplitude of the total field felt by the QD which drives the $|1\rangle \leftrightarrow|4\rangle$ and $|2\rangle \leftrightarrow|3\rangle$ transitions, and reads

$$
E_{\mathrm{QD}}^{(x)}=\frac{1}{\epsilon_{\mathrm{effs}}}\left[E_{c}+\frac{1}{4 \pi \epsilon_{0} \epsilon_{B}} \frac{S_{x} P_{\mathrm{MNP}}^{(x)}}{R^{3}}\right],
$$

where $\epsilon_{\text {effs }}=\left(2 \epsilon_{B}+\epsilon_{s}\right) /\left(3 \epsilon_{B}\right)$, and $S_{x}=-1$ since the electric field $E_{\mathrm{QD}}^{(x)}$ is polarized along the $X$ axis of the hybrid system [see Fig. 1(b)]. The dipole moment $P_{\mathrm{MNP}}^{(x)}$ arises from the charge induced on the surface of the MNP, and depends on the total field due to the QD as ${ }^{34}$

$$
P_{\mathrm{MNP}}^{(x)}=4 \pi \epsilon_{0} \epsilon_{B} q L_{x}^{3} \gamma_{x}\left(\omega_{L}\right) E_{\mathrm{MNP}}^{(x)},
$$

where $E_{\mathrm{MNP}}^{(x)}$ is the slowly varying field amplitude at frequency $\omega_{L}$ felt by the MNP, which is given by

$$
E_{\mathrm{MNP}}^{(x)}=E_{c}+\frac{1}{4 \pi \epsilon_{0} \epsilon_{B}} \frac{S_{x} P_{\mathrm{QD}}^{(x)}}{R^{3}} .
$$

In Eq. (9) the factor $\gamma_{x}\left(\omega_{L}\right)$ corresponds to the polarization of the MNP, which reads as

$$
\gamma_{l}\left(\omega_{L}\right)=\frac{\epsilon_{m}\left(\omega_{L}\right)-\epsilon_{B}}{3 \epsilon_{B}+3 \varsigma_{l}\left(\epsilon_{m}\left(\omega_{L}\right)-\epsilon_{B}\right)}, \quad l=x, y, z,
$$

where $\varsigma_{l}$ is called the depolarization factor of the $\mathrm{MNP},{ }^{35}$ and for a nanospheroid it reduces to

$$
\begin{aligned}
\varsigma_{x} & =\frac{1-\varsigma_{y}}{2}, \quad \varsigma_{z}=\frac{1-\varsigma_{y}}{2}, \\
\varsigma_{y} & =\frac{1}{q^{2}-1}\left[\frac{1}{\sqrt{1-1 / q^{2}}} \ln \left(\frac{1+\sqrt{1-1 / q^{2}}}{1-\sqrt{1-1 / q^{2}}}\right)-1\right] .
\end{aligned}
$$

In the case where the driving field is polarized along the $Y$ axis, the polarization parameter $S_{x}$ in Eqs. (8) and (10) should 
be changed to $S_{y}=-1$, and in addition the polarization of the MNP $\gamma_{x}$ in Eq. (9) should be substituted by $\gamma_{y}$.

The dipole $P_{\mathrm{QD}}^{(x)}$ is expressed via the off-diagonal elements of the density matrix as follows:

$$
P_{\mathrm{QD}}^{(x)}=\mu_{41} \rho_{41}+\mu_{32} \rho_{32} .
$$

In Eq. (10) we have not included the factor $\epsilon_{\text {effs }}$ to account for the screening of the QD dipole field due to the QD dielectric response, since the polarization $P_{\mathrm{QD}}^{(x)}$ already contains this factor as pointed out by Malyshev et al. ${ }^{36}$ Substituting Eqs. (10) and (13) back into Eq. (9) we obtain

$$
\begin{aligned}
P_{\mathrm{MNP}}^{(x)}= & 2 \pi \epsilon_{0} \epsilon_{B} q L_{x}^{3} \gamma_{x}\left(\omega_{L}\right) \\
& \times\left(E_{c}+\frac{S_{x}}{2 \pi \epsilon_{0} \epsilon_{B}} \frac{\mu_{41} \rho_{41}+\mu_{32} \rho_{32}}{R^{3}}\right) .
\end{aligned}
$$

Finally, the slowly varying amplitude of the field in the QD is

$$
\begin{aligned}
E_{\mathrm{QD}}^{(x)}= & \frac{E_{c}}{\epsilon_{\mathrm{effs}}}\left(1+\frac{S_{x} q L_{x}^{3} \gamma_{x}\left(\omega_{L}\right)}{R^{3}}\right) \\
& +\frac{q L_{x}^{3} \gamma_{x}\left(\omega_{L}\right) S_{x}^{2}\left(\mu_{41} \rho_{41}+\mu_{32} \rho_{32}\right)}{2 \pi \epsilon_{0} \epsilon_{B} \epsilon_{\mathrm{effs}} R^{6}} .
\end{aligned}
$$

By introducing Eq. (14) into Eq. (7), the total Hamiltonian of the QD in the dipole approximation may be expressed as follows:

$$
\begin{aligned}
H= & \hbar \sum_{j=1}^{4} \omega_{j} \sigma_{j j}-\hbar\left[\Omega_{c}+G_{c}\left(\rho_{41}+\rho_{32}\right)\right]\left(\sigma_{41}+\sigma_{32}\right) \\
& \times e^{-i \omega_{L} t}+\text { H.c. }
\end{aligned}
$$

where we have introduced the magnitudes $\Omega_{c}$ and $G_{c}$, which account for the effects of both the driving field and the plasmonic interaction, and are explicitly given by

$$
\begin{aligned}
\Omega_{c}^{0} & =\frac{\mu_{41} E_{c}}{2 \hbar \epsilon_{\mathrm{effs}}}, \quad \Omega_{c}=\Omega_{c}^{0}\left[1+\frac{S_{x} q L_{x}^{3} \gamma_{x}\left(\omega_{L}\right)}{R^{3}}\right], \\
G_{c} & =\frac{S_{x}^{2} \mu_{41}^{2} q L_{x}^{3} \gamma_{x}\left(\omega_{L}\right)}{4 \pi \epsilon_{0} \epsilon_{B} \hbar \epsilon_{\mathrm{effs}} R^{6}},
\end{aligned}
$$

where we have assumed that $\mu_{41}=\mu_{32}$. In the above equations, $\Omega_{c}$ is the renormalized Rabi frequency associated with the driving field and the field produced by the induced dipole moment $P_{\mathrm{MNP}}^{(x)}$ of the MNP. Note that $G_{c}$ is a complex quantity. When the laser field is weak, the imaginary part of $G_{c}$ represents the Förster energy transfer rate from the QD to the MNP and therefore contributes to the damping rate of the QD. The real part of $G_{c}$ refers to the redshift of the QD transition caused by the plasmonic interaction. ${ }^{20,37}$

Finally the term $\mathcal{L} \rho$ in Eq. (6) accounts for the spontaneous decay rates of the involved transition which are described by Lindblad terms

$$
\begin{aligned}
\mathcal{L} \rho= & \frac{\gamma_{41}^{p}}{2}\left(\rho \sigma_{44}+\sigma_{44} \rho-2 \sigma_{14} \rho \sigma_{41}\right) \\
& +\frac{\gamma_{42}^{p}}{2}\left(\rho \sigma_{44}+\sigma_{44} \rho-2 \sigma_{24} \rho \sigma_{42}\right) \\
& +\frac{\gamma_{31}^{p}}{2}\left(\rho \sigma_{33}+\sigma_{33} \rho-2 \sigma_{13} \rho \sigma_{31}\right)
\end{aligned}
$$

$$
\begin{aligned}
& +\frac{\gamma_{32}^{p}}{2}\left(\rho \sigma_{33}+\sigma_{33} \rho-2 \sigma_{23} \rho \sigma_{32}\right) \\
& +\frac{\gamma_{21}}{2}\left(\rho \sigma_{22}+\sigma_{22} \rho-2 \sigma_{12} \rho \sigma_{21}\right)
\end{aligned}
$$

Here, $\gamma_{i j}^{p}$ with $i=3,4$ and $j=1,2$ stand for the spontaneous emission decay rates of the QD. These parameters are modified by the plasmonic field of the MNP. The dissipative process described by the term of the Liouvillian with the prefactor $\gamma_{21}$ accounts for the lower levels' dephasing and is assumed to be uncoupled with the localized surface plasmons due to the low values of the Zeeman splitting considered.

We consider that the QD can lose its excitation radiatively by emitting a photon and nonradiatively through the dissipation of currents induced by the QD in the MNP. In order to describe how the spontaneous emissions of the QD's atomic transitions are modified by the plasmonic interaction, we make use of the classical calculations of the fields as shown for example in Ref. 26. For a particle whose size is much smaller than the wavelength, the calculation simplifies because it is sufficient to consider the quasistatic field solution, ${ }^{26}$ in which the QD is treated as a point dipole. It is well known that the decay rate of an electric dipole in free space is

$$
\gamma_{0}=\frac{1}{2} \int_{S} \vec{E}^{0} \wedge \vec{H}^{0} \cdot d \vec{S} \propto \omega_{L}^{3}\left|\vec{d}_{0}\right|^{2}
$$

where $\vec{d}_{0}$ is the dipolar moment of the atom, and $\vec{E}^{0}\left(\vec{H}^{0}\right)$ is the electric (magnetic) field in free space. When a nanoparticle is close to the atom, the radiation power will change since the electric and magnetic fields reflected on the particle act back upon the atom and modify the emission of the atomic dipole. In this new situation the decay rate is

$$
\gamma=\frac{1}{2} \int_{S}\left(\vec{E}^{0}+\vec{E}^{R}\right) \wedge\left(\vec{H}^{0}+\vec{H}^{R}\right) \cdot d \vec{S} \propto \omega_{L}^{3}\left|\vec{d}_{\mathrm{tot}}\right|^{2}
$$

$\vec{E}^{R}\left(\vec{H}^{R}\right)$ being the reflected electric (magnetic) field, and $\vec{d}_{\text {tot }}$ being the total dipole moment of the hybrid system. In view of these considerations, we can compute an enhancement factor for the decay rate as $F_{\text {enh }}=\gamma / \gamma_{0}=\left|\vec{d}_{\text {tot }}\right|^{2} /\left|\vec{d}_{0}\right|^{2}$. This topic has been previously addressed in detail in previous works. ${ }^{26,38}$ The enhancement factor $\left(F_{\text {enh }}\right)$ depends on both the size of the MNP and its relative orientation to the dipolar moments of the QD. The coherent-plasmonic field enhancement factor in the current case, $F_{\text {enh }}^{x}$, reads as [see Eq. (16)]

$$
F_{\mathrm{enh}}^{x} \equiv\left|\frac{\Omega_{c}}{\Omega_{c}^{0}}\right|^{2}=\left|1+\frac{S_{x} q L_{x}^{3} \gamma_{x}\left(\omega_{L}\right)}{R^{3}}\right|^{2}
$$

In the case of considering a driving field polarized along the $Y$ axis the enhancement factor should read as

$$
F_{\mathrm{enh}}^{y}=\left|1+\frac{S_{y} q L_{x}^{3} \gamma_{y}\left(\omega_{L}\right)}{R^{3}}\right|^{2}
$$

In view of the considerations raised when defining the enhancement factor in Eqs. (20) and (21), the radiative decay 
rates of the atomic transitions modified by the coherentplasmonic field enhancement are given by

$$
\gamma_{41}^{p}=\gamma_{32}^{p}=\gamma_{41}^{(0)} F_{\mathrm{enh}}^{x}, \quad \gamma_{42}^{p}=\gamma_{31}^{p}=\gamma_{42}^{(0)} F_{\mathrm{enh}}^{y},
$$

where the superscript index 0 is used to indicate the values of the decay rates in free space. A similar approach to estimate the modification of the decay rates has been used in other works (see for example Refs. 39-44). It is worth noting that, depending on the orientation of the dipole moments of the QD emitter's transitions, the decay rates could become very different from one another, i.e., the value of $F_{\mathrm{enh}}^{y}$ can strongly differ from that of $F_{\text {enh. }}^{x}$. This will result in an anisotropic Purcell factor enhancement which will have important consequences for controlling the time dynamics of this nanoscale hybrid system, and can lead to an acceleration of the spin initialization to the selected state.

From Eqs. (15) and (17), we obtain the following equations of motion for the density matrix elements of the QD in the hybrid system:

$$
\begin{aligned}
\frac{\partial \rho_{41}}{\partial t}= & -\left[\Gamma_{41}-i \Delta_{c}\right] \rho_{41} \\
& +i\left[\Omega_{c}+G_{c}\left(\rho_{41}+\rho_{32}\right)\right]\left(\rho_{11}-\rho_{44}\right), \\
\frac{\partial \rho_{32}}{\partial t}= & -\left[\Gamma_{32}-i\left(\Delta_{c}+2 \Delta_{e}+2 \Delta_{g}\right)\right] \rho_{32} \\
& +i\left[\Omega_{c}+G_{c}\left(\rho_{41}+\rho_{32}\right)\right]\left(\rho_{22}-\rho_{33}\right), \\
\frac{\partial \rho_{44}}{\partial t}= & -\left(\gamma_{41}^{p}+\gamma_{42}^{p}\right) \rho_{44}+i\left[\Omega_{c}+G_{c}\left(\rho_{41}+\rho_{32}\right)\right] \rho_{14} \\
& -i\left[\Omega_{c}^{*}+G_{c}^{*}\left(\rho_{14}+\rho_{23}\right)\right] \rho_{41}, \\
\frac{\partial \rho_{33}}{\partial t}= & -\left(\gamma_{31}^{p}+\gamma_{32}^{p}\right) \rho_{33}+i\left[\Omega_{c}+G_{c}\left(\rho_{41}+\rho_{32}\right)\right] \rho_{23} \\
& -i\left[\Omega_{c}^{*}+G_{c}^{*}\left(\rho_{14}+\rho_{23}\right)\right] \rho_{32}, \\
\frac{\partial \rho_{22}}{\partial t}= & -\gamma_{21} \rho_{22}+\gamma_{32}^{p} \rho_{33}+\gamma_{42}^{p} \rho_{44} \\
& +i\left[\Omega_{c}^{*}+G_{c}^{*}\left(\rho_{14}+\rho_{23}\right)\right] \rho_{32} \\
& -i\left[\Omega_{c}+G_{c}\left(\rho_{41}+\rho_{32}\right)\right] \rho_{23} .
\end{aligned}
$$

We assume the following definitions of the dephasing rates: $\Gamma_{41}=\left(\gamma_{41}^{p}+\gamma_{42}^{p}\right) / 2, \Gamma_{21}=\gamma_{21} / 2$, and $\Gamma_{32}=\left(\gamma_{31}^{p}+\right.$ $\left.\gamma_{32}^{p}+\gamma_{21}\right) / 2=\Gamma_{41}+\Gamma_{21}$. Finally, $\Delta_{c}=\omega_{L}-\omega_{41}$ denotes the optical detuning of the driving field. The population of the ground level $\rho_{11}$ is computed considering a closed system, i.e., $\rho_{11}=1-\rho_{22}-\rho_{33}-\rho_{44}$.

A close inspection of Eq. (23) reveals that the plasmonic interaction manifests in three different ways: The first relies on the plasmon-induced modification of the spontaneous decay rates. The second is related with the enhancement of the Rabi frequency which drives the QD according to the expression given in Eq. (16). The third manifestation of the plasmonic interaction can be formally interpreted as a nonlinear frequency shift in the optical resonance, causing a dynamical detuning and a nonradiative decay rate. For example, in the case of the optical coherence $\rho_{41}$, the dynamical detuning is given by $\operatorname{Re}\left(G_{c}\right)\left(\rho_{11}-\rho_{44}\right)$, whereas the dynamical decay rate (nonradiative decay rate) is given by $\operatorname{Im}\left(G_{c}\right)\left(\rho_{11}-\rho_{44}\right)$. The above mentioned mechanisms strongly depend on the QD-MNP distance $R$. The last two mechanisms have been explored in the context of selective excitonic population in a QD-MNP hybrid system. ${ }^{45}$ In Sec. III we will present the results of spin initialization by means of numerical simulations for the hybrid system and we will discuss the role of the previously mentioned mechanisms.

\section{RESULTS AND DISCUSSION}

We consider the data for the QD reported in Ref. 14 . In particular, the radiative decay rates of the transitions are $\hbar \gamma_{41}^{(0)}=\hbar \gamma_{42}^{(0)}=\hbar \gamma_{32}^{(0)}=\hbar \gamma_{31}^{(0)} \equiv \hbar \gamma_{0}=0.5 \mu \mathrm{eV}$, the hole spin decay rate is $\gamma_{21} \simeq 0.000671 \mu \mathrm{eV}$, and the ground-level Zeeman splitting is $2 \hbar \Delta_{g}=18 \mu \mathrm{eV}$. These data correspond ${ }^{14}$ to a temperature of $4.2 \mathrm{~K}$ and a magnetic field of $2.3 \mathrm{~T}$. We consider an upper value for the Zeeman separation of the excited levels $2 \hbar \Delta_{e} \simeq 10 \mu \mathrm{eV}$. We assume the driving field to be resonant with transition $|1\rangle \leftrightarrow|4\rangle$, i.e., $\Delta_{c}=0(\lambda \simeq$ $947 \mathrm{~nm})$. We also assume that the dielectric constant of the QD is $\epsilon_{s}=12.96$, and the dielectric constant of the host material is $\epsilon_{B}=2.25$. We consider a gold nanorod with semiminor axis $L_{x}=L_{z}=8 \mathrm{~nm}$ and semimajor axis $L_{y}$ scaled with the aspect ratio $q=L_{y} / L_{z}$. The plasma frequency is set to $\hbar \omega_{p}=8.56 \mathrm{eV}$, the high-frequency limit $\epsilon_{\infty}=9.54$, and the damping constant $\hbar \gamma_{p}=0.066 \mathrm{eV}$. With these parameters, the Drude model assumed in Eq. (4) provides a reasonably good fit to tabulated experimental data for photon energies smaller than $3 \mathrm{eV}^{46}$

As a first step in our study we focus our attention on the modifications of the spontaneous emission due to the presence of the MNP. Figures 2(a) and 2(b) show the radiative decay rates $\gamma_{42}^{p}$ and $\gamma_{41}^{p}$ modified by the presence of the MNP as a function of the aspect ratio $q$ for several distances $R$ from the QD center to the MNP center. A close inspection of these figures shows a highly distinctive behavior for the two decay rates: The $\gamma_{41}^{p}$ decay rate shows a monotonous and smooth decrease versus the aspect ratio for all the distances considered, whereas the $\gamma_{42}^{p}$ decay rate presents a dispersive-like behavior with a very large variation in comparison to the value achieved in free space. In fact, $\gamma_{42}^{p}$ presents a range for $q$ where the decay rate is diminished in comparison to that achieved in free space, and more interestingly, another interval for $q$ where this decay rate is strongly enhanced up to a factor larger than $10^{2}$ times the value in free space. In particular, there exists a certain value of the aspect ratio around $q \simeq 4.6$ for which the enhancement is maximized. This anisotropic behavior is associated with the difference between the longitudinal surface plasmon resonance along the $Y$ axis and the transverse surface plasmon resonance along the $X$ axis. This resonant behavior is depicted in Fig. 2(c) where the angular frequency of the localized surface plasmon polaritons (SPPs) along the principal axes of the nanospheroid are plotted versus the aspect ratio $q$ for a selected distance of $R=40 \mathrm{~nm}$. The frequencies of the SPPs are determined by setting to zero the real part part of the denominator of $\gamma_{y}\left(\omega_{L}\right)$ and $\gamma_{x}\left(\omega_{L}\right)$, and solving for $\omega$ (see also Ref. 38). The dotted line is an eye guide which indicates the transition frequency $\omega_{41}(\lambda \simeq 947 \mathrm{~nm})$. A proper selection of the shape factor of the MNP allows for the excitation of the SPPs along the 

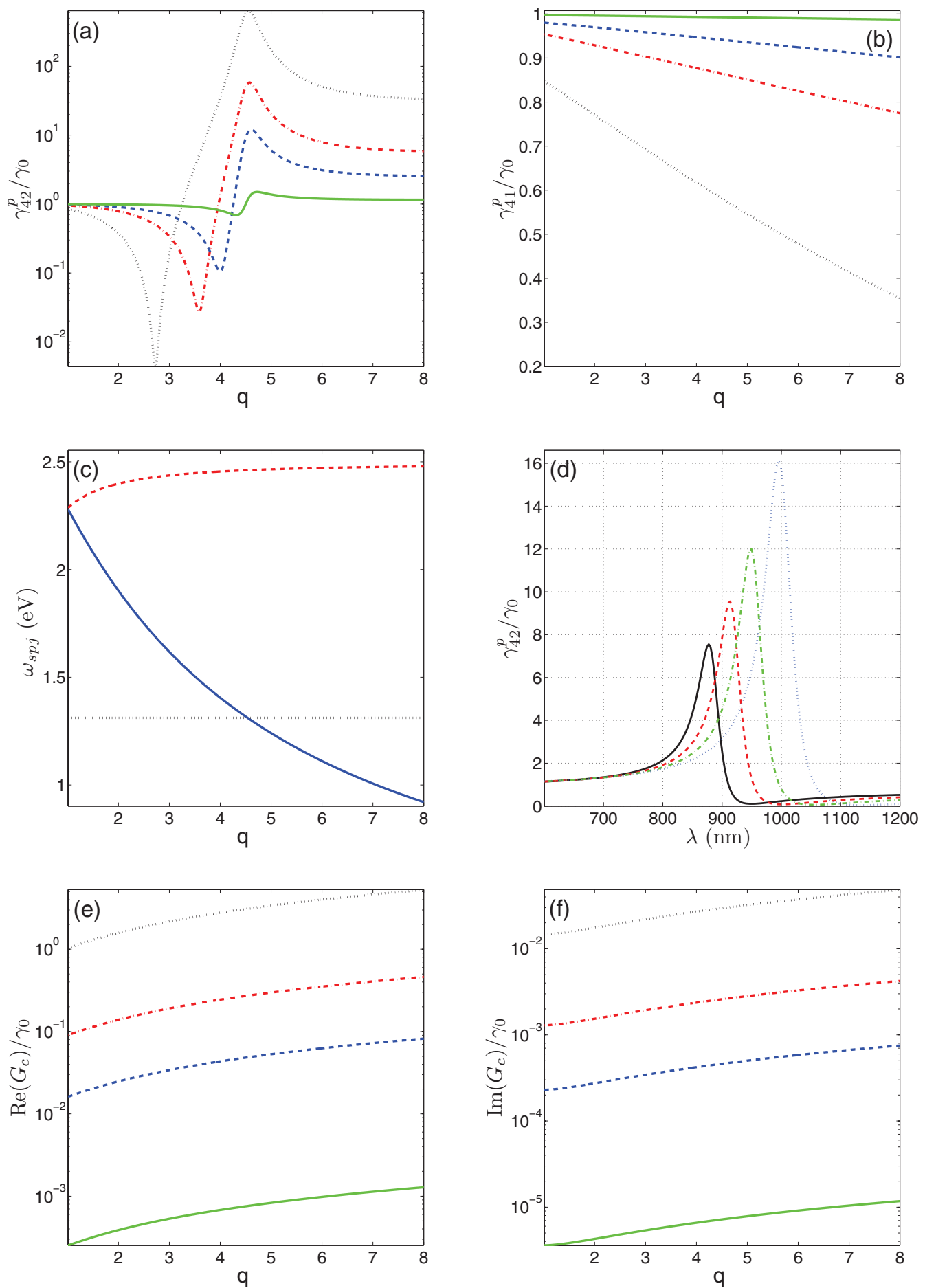

FIG. 2. (Color online) (a),(b) Plasmon modified radiative decay rates of the QD $\gamma_{42}^{p}$ and $\gamma_{41}^{p}$ normalized to $\gamma_{0}$ versus the aspect ratio of the MNP $(q)$ for several distances $R$ from the QD to the MNP: $R=20 \mathrm{~nm}$ (dotted line), $R=30 \mathrm{~nm}$ (dashed-dotted line), $R=40 \mathrm{~nm}$ (dashed line), and $R=80 \mathrm{~nm}$ (solid line). (c) Frequency of the localized plasmon polaritons $\omega_{s p j}(j=y, x)$ along the major axis $Y$ ( $\omega_{\text {spy }}$, solid line) and minor axis $X\left(\omega_{s p x}\right.$, dashed line) in the case with $R=40 \mathrm{~nm}$. The horizontal dotted line indicates the transition frequency $\omega_{41}$. (d) Radiative decay rate $\gamma_{42}^{p}$ normalized to $\gamma_{0}$ versus the wavelength for different values of the aspect ratio $q=4$ (solid line), $q=4.3$ (dashed line), $q=4.6$ (dashed-dotted line), and $q=5$ (dotted line) and in the case with $R=40 \mathrm{~nm}$. (e),(f) Real and imaginary parts of $G_{c}$ normalized to $\gamma_{0}$ versus the aspect ratio $(q)$ for the distances considered in (a), and using the same labels.

$Y$ axis (solid line), whereas those along the $X$ axis remain off resonance (dashed line).

In order to get some further insight into the behavior of the curves depicted in Fig. 2(a), we resort to approximate magnitude $F_{\mathrm{enh}}^{y}$ in the case that the angular frequency of the driving field is on resonance with the atomic transition frequency $\left(\omega_{L}=\omega_{41}\right)$. In the Appendix we show that, when the transision frequency of the QD $\left(\omega_{41}\right)$ is close to the plasmon 
frequency $\omega_{\text {spy }}$, the coherent-plasmonic enhancement factor $F_{\text {enh }}^{y}$ can be simplified to

$$
F_{\mathrm{enh}}^{y} \approx\left|1+q\left(\frac{L_{x}}{R}\right)^{3} \frac{\omega_{\mathrm{spy}}^{2}}{6 \varsigma_{y} \omega_{41}} \frac{\omega_{41}-\omega_{\mathrm{spy}}}{\left(\omega_{41}-\omega_{\mathrm{spy}}\right)^{2}+\left(\gamma_{p} / 2\right)^{2}}\right|^{2},
$$

In the case with $q \in[1,4.6$ ] [see Fig. 2(c)], the surface plasmon frequency along the $Y$ axis is larger than the atomic transition frequency $\left(\omega_{\text {spy }}>\omega_{41}\right)$, thus the second term of the right-hand side of the Eq. (24) takes a negative value, and therefore the enhancement factor $F_{\text {enh }}^{y}$ is less than unity, i.e., the spontaneous emission rate is significantly inhibited, in accordance with the behavior of an emitter inside a cavity. ${ }^{4,47,48}$ On the other hand, in the case where $\omega_{\text {spy }}<\omega_{41}$, which holds in the interval $q \in[4.6,8]$, this term becomes positive, resulting in an enhancement of the spontaneous emission rate $\left(F_{\mathrm{enh}}^{y}>1\right)$. A similar behavior has been found in Refs. 49 and 50. In summary, the MNP acts as a nanoscale cavity which decreases or enhances the strength of the vacuum fluctuations depending on whether $\omega_{\text {spy }}$ lies above or below the atomic transition frequency $\omega_{41}$.

Figure 2(d) shows the radiative decay rate $\gamma_{42}^{p}$ modified by the presence of the MNP as a function of the wavelength of the incident field for different aspect ratios. We have changed the aspect ratio slightly around $q=4.6$ while keeping $R=40 \mathrm{~nm}$. Here we can devise the tunability of the peak wavelength of the longitudinal SPPs in an elongated nanospheroid. Figure 2(d) shows that the larger the aspect ratio, the higher the peak value achieved. In addition, the maximum decay rate shifts to large wavelengths. In summary, all these facts indicate to us that the engineering of the shape and the size of the nanospheroid can be used to enhance on demand the spontaneous emission in a selected atomic transition of the QD. This anisotropic resonant phenomenon is the key idea that allows the selective plasmonic acceleration of the decay of the transition $|2\rangle \leftrightarrow|4\rangle$ in comparison to the almost unaltered transition $|1\rangle \leftrightarrow|4\rangle$. This will result in the acceleration of population transfer within the ground states of the QD. The enhancement of the spontaneous emission of selected decay channels in the current QD-MNP hybrid system mimics the Purcell acceleration achieved in a QD-micropillar cavity in the Voigt geometry considered in Ref. 17.

The anisotropic enhancement of the decay rates of the atomic transitions is accompanied by the simultaneous modification of the nonlinear parameter $G_{c}$ defined in Eq. (16). The terms involving $G_{c}$ in Eq. (23) accounts for the local-field corrections arising from plasmon interaction that takes place due to the proximity between the QD and the MNP. The influence of such local-field corrections has been previously addressed in other hybrid systems (see Ref. 45) in the context of obtaining selective population transfer in a time regime where the effects of the enhancement of the decay rates remained negligible. Here we are interested in a temporal regime where both effects must be considered. To this end, we have plotted in Figs. 2(e) and 2(f) the real and imaginary parts of $G_{c}$ versus the aspect ratio for the same distances as the ones used to produce Fig. 2(a). A close inspection of Figs. 2(e) and 2(f) indicates that the imaginary part of $G_{c}$ is about two orders of magnitude smaller than the real part, thus it is expected that the major influence of the local-field corrections should arise from the dynamical detuning.

After having studied the effect of the MNP on the spontaneous emission and the local-field corrections, now we turn our attention to their impact on the time dynamics of the hybrid system. We have carried out numerical simulations by solving the density matrix equations [Eq. (23)], using a time interval ranging from $t_{0}=0$ to $t_{F}=50 \gamma_{0}^{-1}$, where the spontaneous decay time is $\gamma_{0}^{-1} \simeq 1.3 \mathrm{~ns}$. This time interval allows us to guarantee that a steady state is reached. We also consider standard atomic initial conditions given by $\rho_{11}(0)=1$, and the rest of the elements of $\rho$ equal to zero. In particular, we are interested in the initialization of the spin sate in the QD. The spin state preparation consists of the resonant excitation of $|1\rangle \rightarrow|4\rangle$ transition by the $X$-polarized field. Then, the system can relax to the desired state $|2\rangle$ or to the initial state $|1\rangle$ at different rates $\left(\gamma_{42}^{p} \gg \gamma_{41}^{p}\right)$. The population of the target state $|2\rangle$ increases with a characteristic time $T_{0}$ which is usually related to the spontaneous decay rate of the exciton. The fidelity of the state preparation is commonly defined as $F=\langle\Psi|\rho| \Psi\rangle,|\Psi\rangle=|2\rangle$ being the target state, and $\rho$ stands for the density matrix of the system. The initial value for $F$ in the current situation is 0 and should be 1 in the case of maximum purification of the state $(F=1)$ where the final state should be $|2\rangle$. We can define the initialization error $\epsilon \equiv 1-F$ as the deviation from unity of the fidelity achieved.

We will analyze the time dynamics of the population of the target state $\rho_{22}(t)$. The parameter space of the hybrid system is wide enough to explore it in detail. Thus, we resort to analyze two physical situations which allow us to isolate the effects of the enhancement of the spontaneous decay rates from those arising from local-field corrections. The results of numerical simulations will be presented in Secs. III A and III B, respectively.

\section{A. Initialization in the regime of low dynamical detuning}

We have selected the distance $R=40 \mathrm{~nm}$ to analyze the time dynamics of the population of the target state $\rho_{22}(t)$. The aspect ratio is set to $q=4.6$ which results in $\gamma_{42}^{p} \approx 12 \gamma_{0}$ [very close to the maximum of the dashed line in Fig. 2(a)] and $\gamma_{41}^{p} \approx 0.94 \gamma_{0}$. This choice results in $\operatorname{Re}\left(G_{c}\right) \approx 0.049 \gamma_{0}$, and $\operatorname{Im}\left(G_{c}\right) \approx 0.0005 \gamma_{0}$ [see Figs. 2(e) and 2(f)], so that the terms involving $G_{c}$ in Eq. (23) do not play a significant role in the time dynamics of the system. These data indicate that the asymmetric enhancement of the decay rates is significative while the local field effects remain negligible; i.e., we deal with the so-called regime of low dynamical detuning.

Figures 3(a)-3(c) show the population of the target state $|2\rangle$ as a function of time for several values of the Rabi frequency $\Omega_{c}^{0}$ in the presence and in the absence of the MNP. The vertical solid line is set to $t=\gamma_{0}^{-1}$ and serves as an eye guide to analyze the speed-up of the transfer of population to level $|2\rangle$. The results obtained for a low value of the Rabi frequency of the driving field $\left(\Omega_{c}^{0}=0.5 \gamma_{0}\right)$ are plotted in panel (a), and they show that the time elapsed to reach steady state is large. The main effect of the MNP in this case is to slow down the population transfer to the target level $|2\rangle: \rho_{22}(t=5 \mathrm{~ns}) \simeq 0.41$ in the absence of the MNP, whereas $\rho_{22}(t=5 \mathrm{~ns}) \simeq 0.21$ when the MNP is present. 

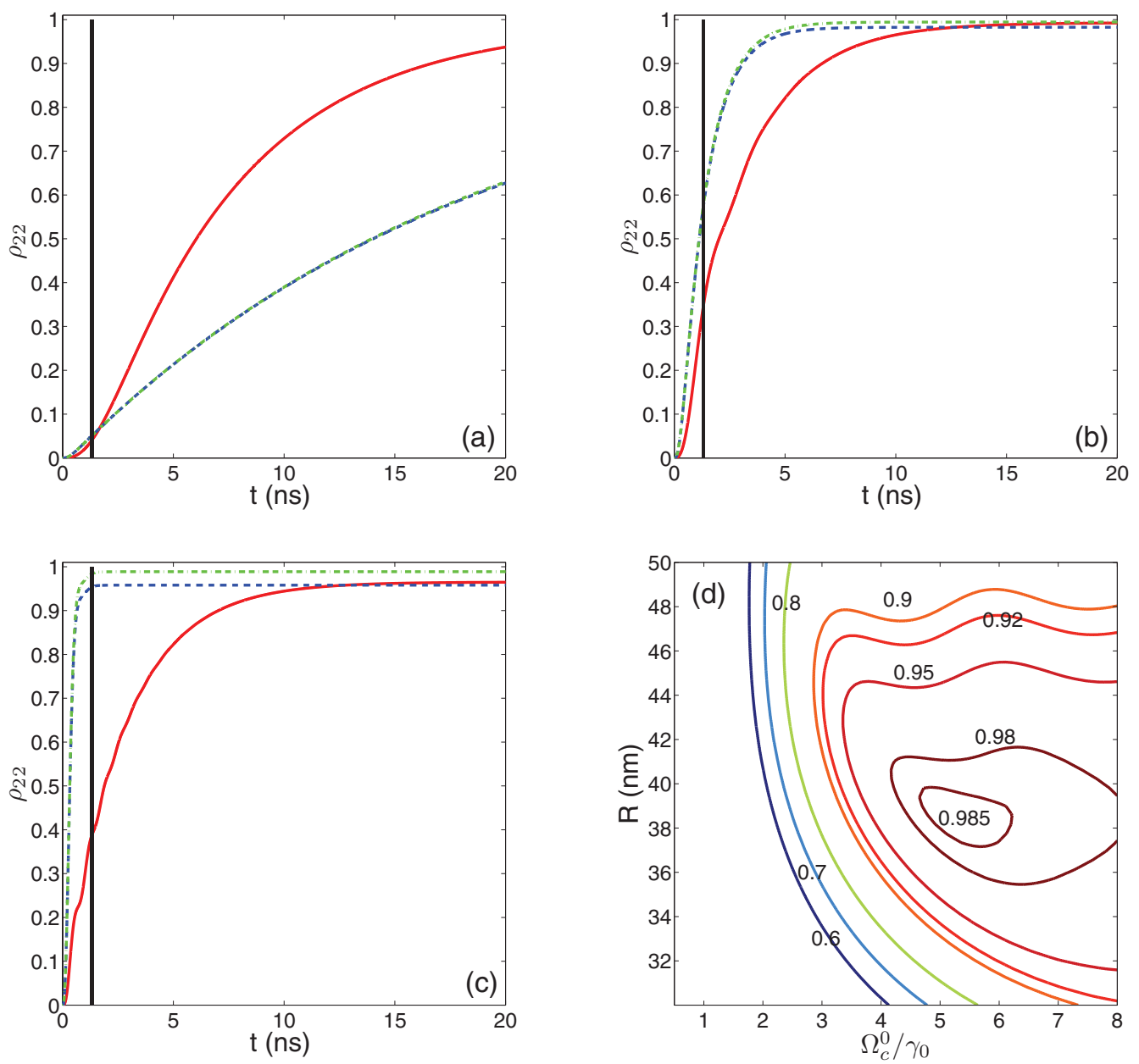

FIG. 3. (Color online) Time evolution of population $\rho_{22}(t)$ for different Rabi frequencies of the driving field: (a) $\Omega_{c}^{0}=0.5 \gamma_{0}$, (b) $\Omega_{c}^{0}=2 \gamma_{0}$, $\Omega_{c}^{0}=6 \gamma_{0}$. Solid line corresponds to the case without the MNP and the other two curves correspond to $R=40 \mathrm{~nm}$. The magnetic field is set to $B_{x}=2.3 \mathrm{~T}$ (solid and dashed line) and $B_{x}=4.6 \mathrm{~T}$ (dashed-dotted line). The parameters of the MNP are $L_{z}=8 \mathrm{~nm}$ and $q=4.6$. In (a) $-(\mathrm{d})$ the vertical thick solid line represents $t=\gamma_{0}^{-1}$. (d) Contour plot of the population of the target level at the instant of time $t=t_{F}$ versus the distance $R$ and the Rabi frequency $\Omega_{c}^{0}$ for a magnetic field $B_{x}=4.6 \mathrm{~T}$.

In the case of using a large value of the Rabi frequency $\left(\Omega_{c}^{0}=2 \gamma_{0}\right)$, a dramatic change on the dynamics takes place: The population transfer becomes more efficient and faster than in the previous case [compare the dashed line in Fig. 3(b) to that in Fig. 3(a)]. Thus, the plasmon interaction speeds up the spin hole initialization without appreciably affecting the fidelity. A further increase of the Rabi frequency $\left(\Omega_{c}^{0}=6 \gamma_{0}\right)$ leads to a very fast initialization speed, although the final value of the population achieved in level $|2\rangle$ is slightly depleted [see dashed line in Fig. 3(c)]. This population depletion arises from the off-resonant optical transition $|2\rangle \leftrightarrow|3\rangle$ which pumps back population from level $|2\rangle$ to level $|1\rangle$. This adverse effect has been previously mentioned in Ref. 15. The unfavorable effects of this off-resonant optical transition can be minimized by increasing the magnetic field, since it will result in a large value for the upper levels' splitting and, consequently, transition $|2\rangle \leftrightarrow|3\rangle$ will become further detuned from the laser field. This effect can be seen when comparing the dashed and dashed-dotted lines in Figs. 3(b) and 3(c), which have been obtained for two different values of $B_{x}$. We conclude that using larger values of magnetic field raises of the efficiency of population transfer to the target level without affecting the speed-up of the hole spin initialization.

For a deeper insight into the problem, we resort to obtain an analytical estimation of the time required to reach the steady state; that is, the spin initialization time $T_{0}$. To this end, we neglect the effects of the off-resonant transition $|2\rangle \leftrightarrow|3\rangle$, the decay rate between ground levels $\left(\gamma_{21} \simeq 0\right)$, and the terms involving $G_{c}$. We also consider $\Omega_{c} \simeq \Omega_{c}^{0}\left(F_{\text {enh }}^{x} \simeq 1\right)$. In this limit the density matrix equations (23) reduce to only three elements: $\rho_{41}, \rho_{44}$, and $\rho_{22}$, and we now deal with a three-level $\Lambda$-type system obeying the following linearized equations of motion:

$$
\begin{aligned}
\frac{\partial \rho_{41}^{I}}{\partial t} & =-\Gamma_{41} \rho_{41}^{I}-\Omega_{c}^{0}\left(\rho_{22}+2 \rho_{44}-1\right), \\
\frac{\partial \rho_{44}}{\partial t} & =-\left(\gamma_{0}+\gamma_{42}^{p}\right) \rho_{44}+2 \Omega_{c}^{0} \rho_{41}^{I}, \\
\frac{\partial \rho_{22}}{\partial t} & =\gamma_{42}^{p} \rho_{44},
\end{aligned}
$$

where $\rho_{41}^{I}=\operatorname{Im}\left(\rho_{41}\right)$ is the imaginary part of $\rho_{41}$. The dynamics of the system is characterized through the 
eigenvalues of the matrix of the equations of motion (25). The eigenvalues are the roots of the following equation:

$$
\begin{aligned}
& \lambda^{3}+\frac{3\left(\gamma_{0}+\gamma_{42}^{p}\right)}{2} \lambda^{2}+\left[\frac{\left(\gamma_{0}+\gamma_{42}^{p}\right)^{2}}{2}+4\left(\Omega_{c}^{0}\right)^{2}\right] \lambda \\
& +2 \gamma_{42}^{p}\left(\Omega_{c}^{0}\right)^{2}=0 .
\end{aligned}
$$

The initialization time is obtained through the smallest eigenvalue, i.e., $T_{0}^{-1}=\min \left|\operatorname{Re}\left(\lambda_{j}\right)\right|$. At low values of the driving field $\left(\Omega_{c}^{0}<\gamma_{0}, \gamma_{42}^{p}\right)$ the characteristic time is estimated to be

$$
T_{0} \simeq \frac{\left(\gamma_{0}+\gamma_{42}^{p}\right)^{2}}{4 \gamma_{42}^{p}\left(\Omega_{c}^{0}\right)^{2}}
$$

The most interesting situation occurs for large driving fields $\left(\Omega_{c}^{0}>\gamma_{0}, \gamma_{42}^{p}\right)$ where high fidelity is achieved. In this strong driving field regime, the characteristic time reads

$$
T_{0} \simeq \frac{2}{\gamma_{42}^{p}}\left(1+\frac{\gamma_{0}\left(2 \gamma_{0}+\gamma_{42}^{p}\right)}{4^{2}\left(\Omega_{c}^{0}\right)^{2}}\right) .
$$

In the case of large values of the Rabi frequency $\Omega_{c}^{0}$, the characteristic time tends to $T_{0} \simeq 2 / \gamma_{42}^{p}=\left(2 \gamma_{0}^{-1}\right) / F_{\text {enh }}^{y}$. This result confirms that the reduction of the initialization time is directly related to the decay rate enhancement induced by the plasmonic interaction.

Let us estimate the initialization time $T_{0}$ from Eq. (27) for $\Omega_{c}^{0} \simeq 0.5 \gamma_{0}$ used to produce Fig. 3(a). The results are $T_{0} \simeq 6.4 \mathrm{~ns}$ without the MNP, and $T_{0} \simeq 16.7 \mathrm{~ns}$ with the MNP, and they confirm the slow-down of the population dynamics displayed in Fig. 3(a). In the case with $\Omega_{c}^{0} \simeq 6 \gamma_{0}$ used to produce Fig. 3(c), the estimation for the initialization time based in Eq. (28) produces $2.65(0.23) \mathrm{ns}$ in the absence (presence) of the MNP. Thus, the characteristic time is reduced by an order of magnitude, in agreement with the enhancement of $\gamma_{42}^{p}\left(F_{\mathrm{enh}}^{y} \simeq 12\right)$. We conclude that the enhancement of the decay rate $\gamma_{42}^{p}$ induced by the MNP leads to a shortening of the characteristic time for an appropriate selection of the Rabi frequency of the driving field, in agreement with the results depicted in Figs. 3(b) and 3(c).

The population transferred to the target level for a fixed instant of time depends on both the distance $R$ and the Rabi frequency $\Omega_{c}^{0}$ as displayed in Fig. 3(d). This picture reveals the interplay between both magnitudes in the current system, and indicates that there exists a region on the parameter space of the system which allows for a high-fidelity spin initialization greater than 0.98 which does not require a precise relative positioning of the MNP relative to the QD or a precise value of the Rabi frequency of the driving field. This point is essential to address the experimental implementation of this hybrid system.

Now we turn our attention to consider an input pulse to drive the hybrid system. To this end, we consider a pulse with a Gaussian shape,

$$
\Omega_{c}^{0}(t)=\frac{\Omega_{c, a}}{\sqrt{2 \pi \sigma^{2}}} e^{-\left(t-t_{p}\right)^{2} /\left(2 \sigma^{2}\right)},
$$

where $\sqrt{2} \sigma$ is the $1 / e$ half-width, $\Omega_{c, a}$ is a dimensionless peak amplitude, and the pulse is centered at $t_{p}$. This definition of the pulse envelope allows us to specify the pulse area merely by the peak amplitude $\Omega_{c, a}$. In what follows we consider a pulse width $\sigma=\gamma_{0}^{-1}$. Figure 4(a) displays the value of population of the target level at $t=t_{p}+\sigma$ as a function of the pulse area. There we observe that for a pulse area larger than $\Omega_{c, a}^{0}=2.6 \pi$ the population transfer exhibits a saturating behavior. Thus, we select this value in order to explore the influence of SPPs on the time dynamics. Figure 4(b) shows the time evolution of the population of the level $|2\rangle$ in the presence and in the absence of the MNP, and it confirms that the MNP allows the achievement of a large and fast population transfer from level $|1\rangle$ to $|2\rangle$ (solid line) in comparison to the case without the MNP (dashed line). The spin state preparation corresponds to a spin temperature of $0.04 \mathrm{~K}$ that will be achieved $5 \mathrm{~ns}$ after the optical pulse should impinge the system.

Figure 4(c) shows the population of level $|2\rangle$ as a function of the aspect ratio $q$. The maximum value of the fidelity that can be achieved is close to 1 for values of $q$ around 4.6, where the decay $\gamma_{42}^{p}$ reaches the largest value [see dashed line in Fig. 2(a)]. It is worth mentioning that the proper selection of the aspect ratio is essential to achieve a high fidelity: Note that for values close to $q \approx 4$ the population of the target state does not experience a significant change from its initial value. One may expect that the maximum transfer of population from level $|1\rangle$ to $|2\rangle$ should take place for the aspect ratio $q=4.6$, where $\gamma_{42}^{p}$ reaches its maximum value [see solid line in Fig. 2(a)]. However, this enhancement of the decay rate also modifies the decay rate of the transition $|1\rangle \leftrightarrow|3\rangle\left(\gamma_{31}^{p}=\gamma_{42}^{p}\right)$, and, in addition, the laser field $E_{c}$ also drives transition $|2\rangle \leftrightarrow|3\rangle$. Thus level $|3\rangle$ is slightly populated by the laser field and its population returns back to level $|1\rangle$ in an accelerated way. This mechanism diminishes the efficacy of the population transfer to the target level $|2\rangle$. Thus, the aspect ratio for which the maximum transfer of population is achieved will be that which optimizes these two competing phenomena. This effect is shown in the inset of Fig. 4(c) when considering the driving pulse as in Fig. 4(b). Numerical analysis reveals that the aspect ratio which maximizes the fidelity is achieved at $q=4.85$.

The initialization error as a function of the external magnetic field $B_{x}$ is depicted in Fig. 4(d) for different values of the aspect ratio. This figure shows that the increase of the magnetic field reduces the magnitude of $\epsilon$. This behavior can be easily understood by considering that the increase of $B_{x}$ produces an enlargement of both the lower levels' splitting $\left(\omega_{21}\right)$ and that of the upper levels $\left(\omega_{43}\right)$. Consequently, the transition $|2\rangle \leftrightarrow|3\rangle$ becomes further detuned, which causes level $|3\rangle$ to become nearly uncoupled from the evolution of the system, i.e., the four-level system reduces to a $\Lambda$-type atom with levels $|1\rangle,|4\rangle$, and $|2\rangle$ for large values of $B_{x}$. A similar behavior has been implicitly assumed to take place in Refs. 15 and 17.

\section{B. Initialization in the regime of large dynamical detuning}

Here we are interested in the situation where both the enhancement of the decay rate $\gamma_{42}^{p}$ and the local field become significant. This requires that we should search for a region of the parameter space of the hybrid system in which $\gamma_{42}^{p}$ and $G_{c}$ are simultaneously enlarged. The aspect ratio $q$ of the MNP is essential to achieve this condition. 

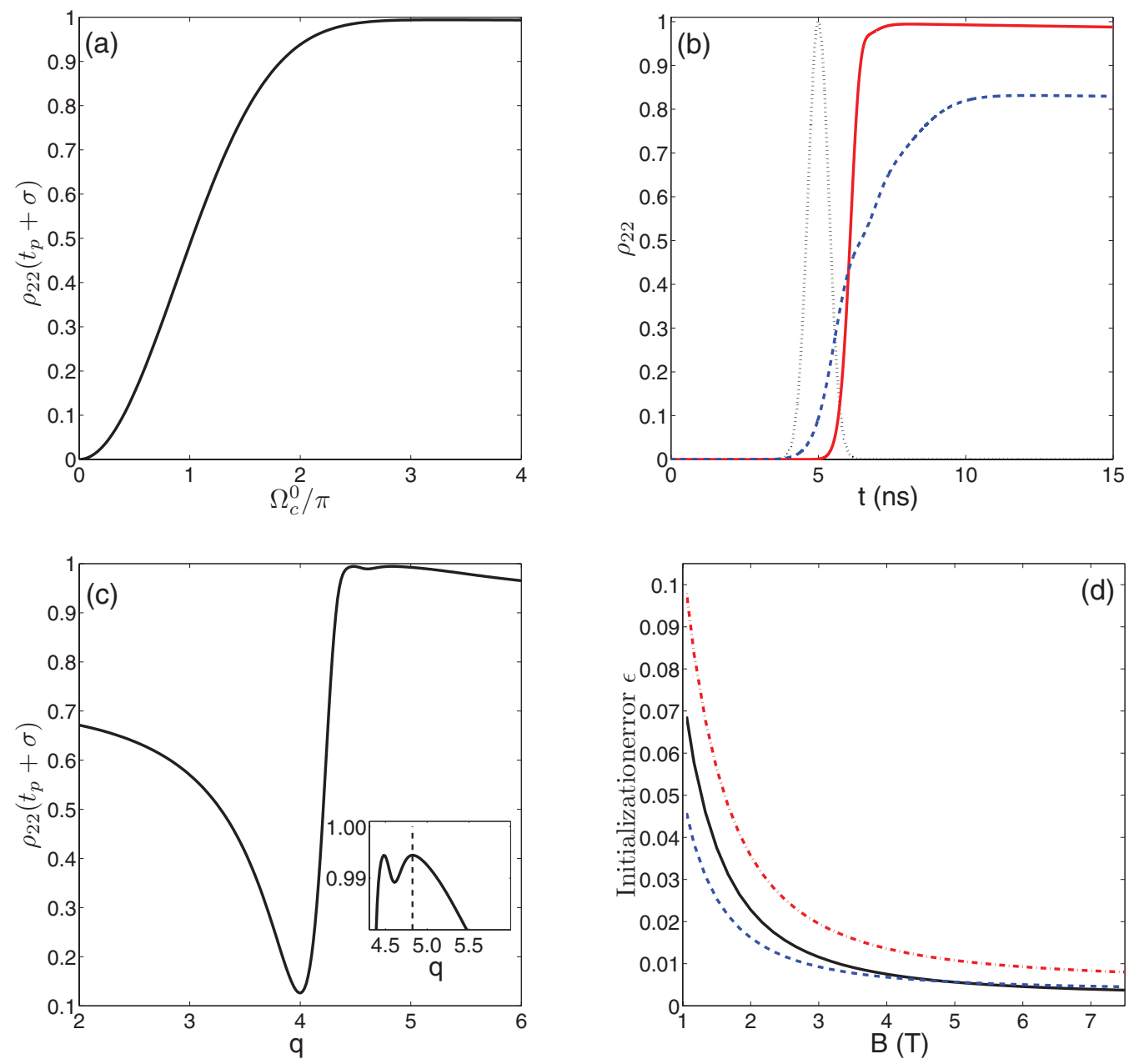

FIG. 4. (Color online) (a) Population of the target state $|2\rangle$ at the instant of time $t=t_{p}+\sigma$ as a function of the area of the input pulse. (b) Time evolution of population $\rho_{22}$ in the presence (solid) and in the absence of MNP (dashed). The black dotted line is the normalized input pulse. (c) Population at the instant of time $t=t_{p}+\sigma$ as a function of the aspect ratio $q$. The vertical dashed line in the inset indicates the value of $q=4.85$ which provides the maximum fidelity achieved. (d) Initialization error $\epsilon$ as a function of the external magnetic field $B_{x}$ for different values of the aspect ratio $q=4.5$ (solid line), $q=4.6$ (dashed-dotted line), and $q=5$ (dashed line). In (a), (b), and (d) $q=4.6 . B_{x}=2.3 \mathrm{~T}$ in (a) and $B_{x}=4.6 \mathrm{~T}$ in (b) and (c). In (b)-(d) $\Omega_{c, a}=2.6 \pi . R=40 \mathrm{~nm}$ and $L_{z}=8 \mathrm{~nm}$ in all panels.

Figure 5 presents the variation of these magnitudes versus the distance $R$ for several values of the aspect ratio. Panel (a) in Fig. 5 presents the enhancement of the decay rate of the $|4\rangle \leftrightarrow$ |2) transition and shows a monotonous decrease of $\gamma_{42}^{p}$ versus distance $R$. In the case where $q=3.2$ the values for $\gamma_{42}^{p}$ become less than unity for $R>19.5 \mathrm{~nm}$, which indicates that for such distances the dynamics should be slowed down due to the plasmonic interaction. For other values of $R$ the enhancement of the $\gamma_{42}^{p}$ decay rate is also accompanied by a minor modification of $\gamma_{41}^{p}$ which is nearly independent of the aspect ratio (not shown). The behavior of the real part of $G_{c}$ depicted in Fig. 5(b) also presents a monotonous decrease versus the distance $R$. The aspect ratio has a negligible influence on $G_{c}$ in view of the fact that the three curves in Fig. 5(b) remain close together. The relative value of the quotient $\operatorname{Re}\left(G_{c}\right) / \gamma_{42}^{p}$ is presented in Fig. 5(c) and serves as an indication that the terms involving $G_{c}$ in the equation of motion (23) will compete with the linear terms in the case of dealing with small values of $R$. Thus, the major influence of the local field on the time dynamics should be expected when considering low values of $R$.
We have selected two points in the parameter space $(R, q)$ of the system which share the same enhancement for the decay rate $\gamma_{42}^{p}$ : The first one is for a distance of $R=16 \mathrm{~nm}$ and the second for a distance $R=40 \mathrm{~nm}$. The latter is the one considered in the previous subsection, and for an aspect ratio $q=4.6$ it was shown that $\gamma_{42}^{p}(q=4.6, R=40 \mathrm{~nm}) \approx 12 \gamma_{0}$. We have determined the aspect ratio which should produce the same enhancement for the other distance considered $(R=16 \mathrm{~nm})$, which was found to be $q=3.35$, i.e., $\gamma_{42}^{p}(q=3.35, R=16 \mathrm{~nm}) \approx 12 \gamma_{0}$. We have solved the equations of motion (23) for these two pairs of points $(R, q)$ and different values of $\Omega_{c}^{0}$. In the case where $R=16 \mathrm{~nm}$ we obtain $G_{c}=(9.11+i 0.09) \gamma_{0}$, whereas in the case with $R=40 \mathrm{~nm}$ we obtained $G_{c}=(0.049+i 0.0005) \gamma_{0}$. The results are presented in Fig. 6 and they indicate that in all cases the achievement of the steady state of $\rho_{22}(t)$ for the shortest distance is slowed down in comparison to the largest one, i.e., no acceleration is found in the case of the smallest value for $R$. These results contrast with those found when analyzing the time dynamics in the case of negligible local fields presented in 

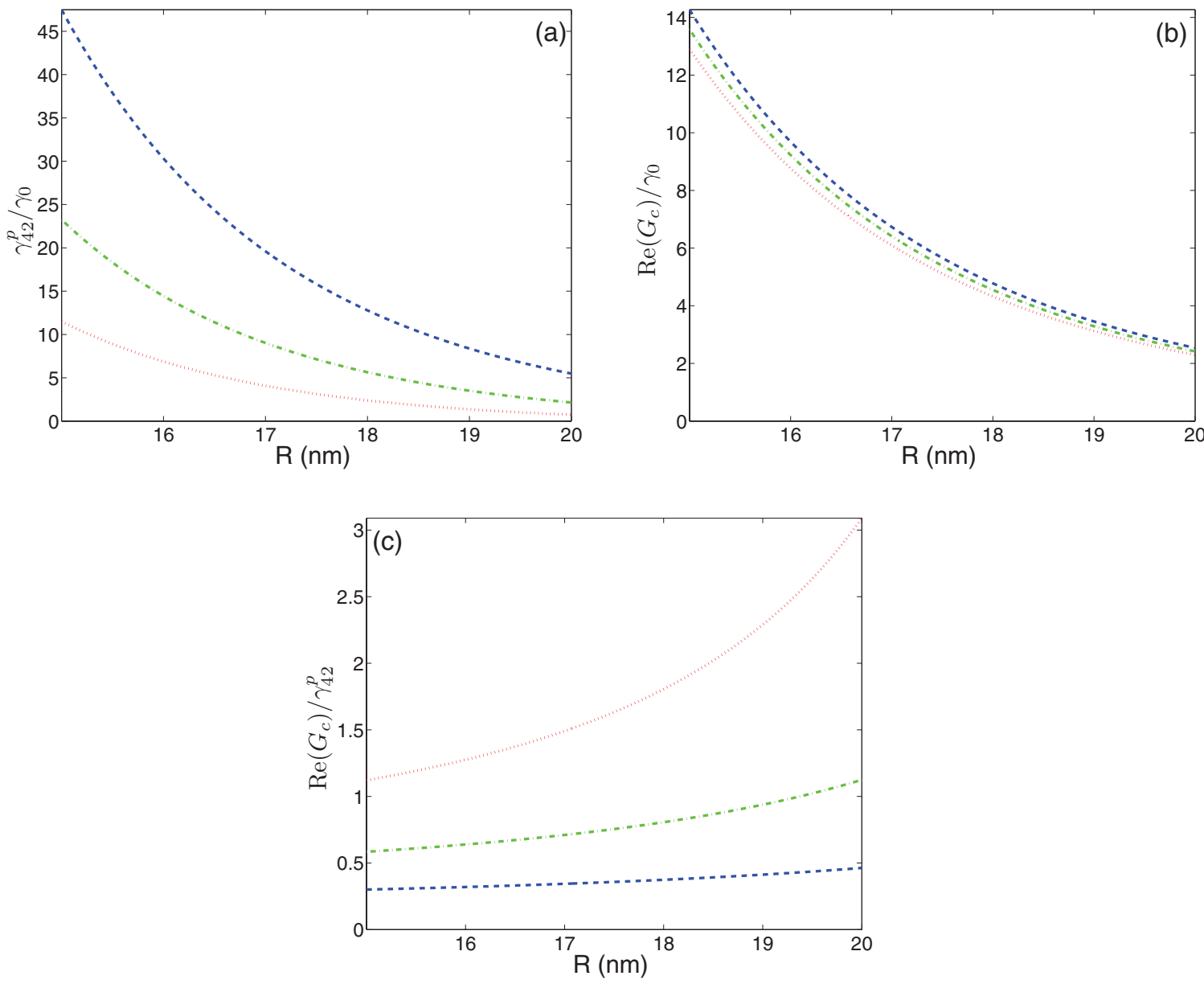

FIG. 5. (Color online) (a) Plasmon modified radiative decay rates of the QD $\gamma_{42}^{p}$ normalized to $\gamma_{0}$ versus the distance $R$. (b) Real part of $G_{c}$ normalized to $\gamma_{0}$ versus the distance $R$. (c) Magnitude $\mathcal{R} e\left(G_{c}\right) / \gamma_{42}^{p}$ versus the distance $R$. The values of the aspect ratio of the MNP $q$ are $q=3.2$ (dotted line), $q=3.4$ (dashed-dotted line), $q=3.6$ (dashed line).

Figs. 3(a)-3(c). The lengthening of the time required to achieve a steady state is fully attributable to the local fields arising from the plasmonic interaction between the QD and the MNP. This result is in accordance with those reported by Jyotsna and Agarwal $^{51}$ in a dense collection of $\Lambda$-type atoms, and those reported by Calderón et al. ${ }^{52}$ in a dense collection of $V$-type atoms. In both cases the effect was shown that the local field causes an increase in the time required to achieve a steady state.

Finally, it should be stated that, although speculative, the hybrid system here investigated can be implemented in realistic QD-MNP systems. Recently, hybrid structures consisting of self-assembled QDs have been grown and covered with metall nanocrystals. ${ }^{53}$ In addition, we would like to draw attention to very recent works where the controlled coupling of a single epitaxial quantum dot to a plasmonic nanoantenna has been demonstrated ${ }^{54,55}$ Thus, the structure modeled here could be fabricated using available nanotechnologies for the growth and the precise positioning of the involved elements.

\section{CONCLUSIONS}

In this work we present a description of the exciton-plasmon interaction in a QD-MNP hybrid system intended for obtaining high fidelity spin preparation. The QD is modeled as a four-level-like atomic system and the MNP is considered to be a nanospheroid. We analyze how localized surface charge oscillations in the MNP modify the decay rates of the QD depending on the aspect ratio of the MNP and the distance between the QD and MNP $R$. Based on the fact that the frequencies of the SPPs are very different along the two principal axes, the decay rate of the atomic transitions parallel to the MNP's major axis would be much larger than those parallel to the corresponding minor axis. We show that the anisotropic acceleration of the decay rates allows for a fast spin initialization in the QD using a short optical pulse. The hole spin is predicted to be successfully cooled from 5 to $0.04 \mathrm{~K}$ at a magnetic field of $4.6 \mathrm{~T}$ applied in the Voigt geometry. The characteristic time and the fidelity are analyzed as a function of the MNP aspect ratio, the applied external magnetic field, and the Rabi frequency of the driving field. We have also demonstrated that, for distances where large dynamical detuning occurs due to the MNP coupling, a lengthening of the time required for spin state initialization takes place.

The current approach represents a fast way to obtain the spin initialization with high fidelity, which is required for quantum information processing, while taking advantage of a positively charged QD where the dephasing due to nuclear spins is minimized. 

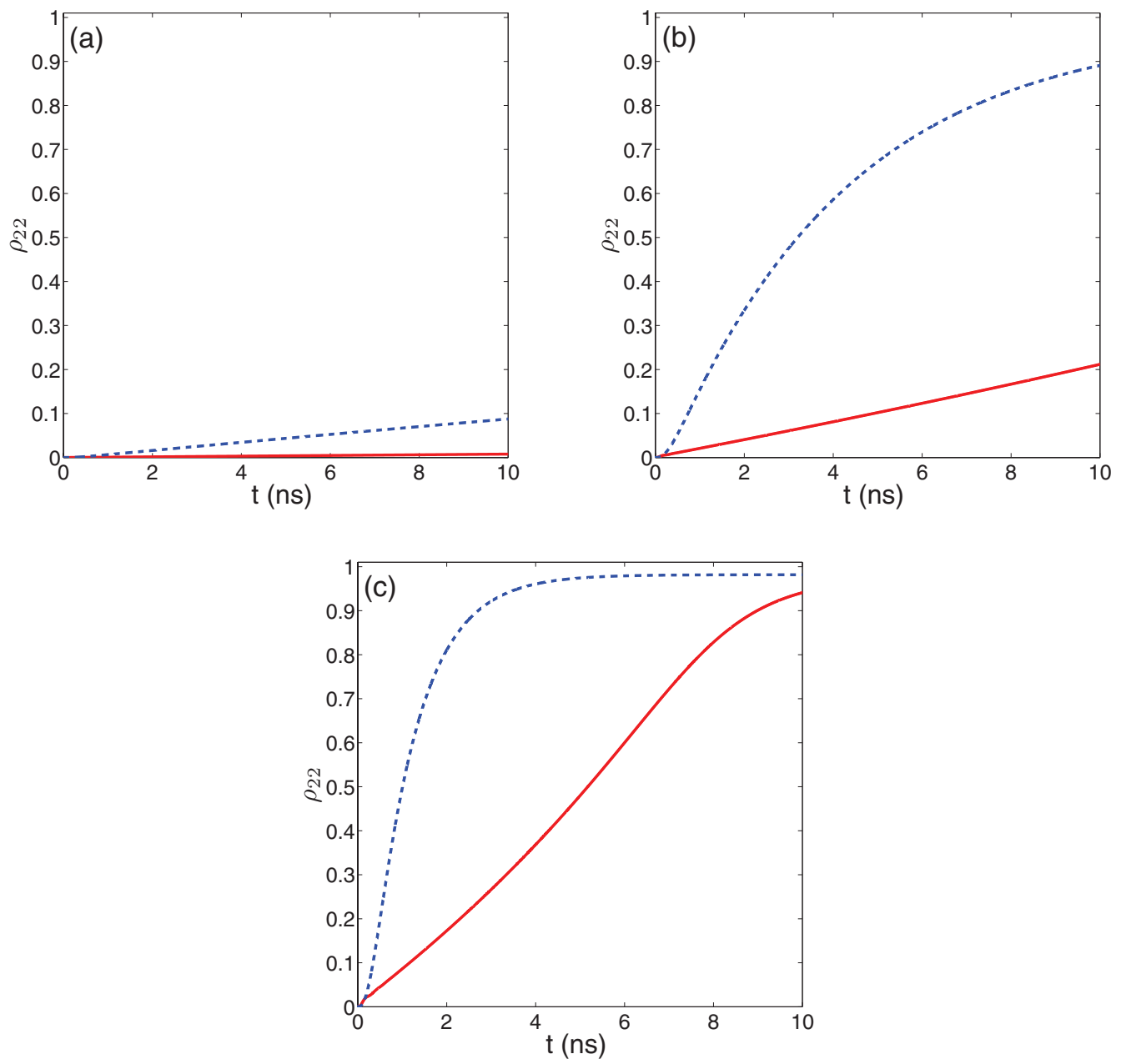

FIG. 6. (Color online) Time evolution of $\rho_{22}(t)$ in the case of $(R, q)=(16 \mathrm{~nm}, 3.35)$ (solid line) and $(R, q)=(40 \mathrm{~nm}, 4.6)($ dashed line). The Rabi frequencies are $\Omega_{c}^{0}=0.2 \gamma_{0}$ in (a), $\Omega_{c}^{0}=\gamma_{0}$ in (b), $\Omega_{c}^{0}=2 \gamma_{0}$ in (c).

\section{ACKNOWLEDGMENTS}

This work has been supported by Project No. FIS201022082 (MCINN) from Spain. The authors want to express their gratitude to J. D. Cox for his invaluable help in revising the manuscript.

\section{APPENDIX: MODIFICATION OF THE SPONTANEOUS EMISSION RATES OF AN EMITTER CLOSE TO A MNP}

When plugging Eq. (4) into Eq. (11) for the case with $l=y$, we attain the expression for the MNP polarizability along the $Y$ axis

$$
\gamma_{y}=\frac{1}{3} \frac{\left(\epsilon_{\infty}-\epsilon_{B}\right)\left(\omega_{L}^{2}+i \gamma_{p} \omega_{L}\right)-\omega_{p}^{2}}{\left[\epsilon_{\infty}+\left(1 / \varsigma_{y}-1\right) \epsilon_{B}\right]\left(\omega_{L}^{2}+i \gamma_{p} \omega_{L}\right)-\omega_{p}^{2}}
$$

The plasmon frequency $\omega_{\text {spy }}$ is obtained by setting to zero the real part of the denominator in Eq. (A1), and reads

$$
\omega_{\mathrm{spy}} \simeq \frac{\omega_{p}}{\sqrt{\epsilon_{\infty}+\left(1 / \varsigma_{y}-1\right) \epsilon_{B}}} .
$$

Taking into account the result in Eq. (A2), neglecting the imaginary part of the numerator, and assuming $\omega_{L}+\omega_{\text {spy }} \approx$ $2 \omega_{L}$, we can rewrite Eq. (A1) as

$$
\gamma_{y} \approx-\frac{\omega_{\mathrm{spy}}^{2}}{6 \varsigma_{y} \omega_{L}} \frac{\omega_{L}-\omega_{\mathrm{spy}}}{\left(\omega_{L}-\omega_{\mathrm{spy}}\right)^{2}+\left(\gamma_{p} / 2\right)^{2}} .
$$

Plugging these approximations back into Eq. (11), we attain Eq. (24).
${ }^{1}$ J. M. Elzerman, R. Hanson, L. H. Willems van Beveren, B. Witkamp, L. M. K. Vandersypen, and L. P. Kouwenhoven, Nature (London) 430, 431 (2004).
${ }^{2}$ D. Heiss, S. Schaeck, H. Huebl, M. Bichler, G. Abstreiter, J. J. Finley, D. V. Bulaev, and D. Loss, Phys. Rev. B 76, 241306R (2007). 
${ }^{3}$ A. Zrenner E. Beham, S. Stufler, F. Findeis, M. Bichler, and G. Abstreiter, Nature 418, 612 (2002).

${ }^{4}$ X. Li, Y. Wu, D. Steel, D. Gammon, T. H. Stievater, D. S. Katzer, D. Park, C. Piermarocchi, and L. J. Sham, Science 301, 809 (2003).

${ }^{5}$ A. Imamoglu, D. D. Awschalom, G. Burkard, D. P. DiVincenzo, D. Loss, M. Sherwin, and A. Small, Phys. Rev. Lett. 83, 4204 (1999). ${ }^{6}$ G. Burkard, D. Loss, and D. P. DiVincenzo, Phys. Rev. B 59, 2070 (1999).

${ }^{7}$ A. C. Johnson, J. R. Petta, J. M. Taylor, M. D. Lukin, C. M. Marcus, M. P. Hanson, and A. C. Gossard, Nature (London) 435, 925 (2005). ${ }^{8}$ W. A. Coish and D. Loss, Phys. Rev. B 70, 195340 (2004).

${ }^{9}$ A. Imamoglu, E. Knill, L. Tian, and P. Zoller, Phys. Rev. Lett. 91, 017402 (2003).

${ }^{10}$ C. W. Lai, P. Maletinsky, A. Badolato, and A. Imamoglu, Phys. Rev. Lett. 96, 167403 (2006).

${ }^{11}$ D. D. Awschalom and J. M. Kikkawa, Phys. Today 52, 33 (1999).

${ }^{12}$ M. Atatüre, J. Dreiser, A. Badolato, A. Högele, K. Karrai, and A. Imamoglu, Science 312, 551 (2006).

${ }^{13}$ D. V. Bulaev and D. Loss, Phys. Rev. Lett. 95, 076805 (2005).

${ }^{14}$ D. Brunner, B. D. Gerardot, P. A. Dalgarno, G. Wüst, K. Karrai, N. G. Stoltz, P. M. Petroff, and R. J. Warburton, Science 325, 70 (2009).

${ }^{15}$ C. Emary, X. Xu, D. G. Steel, S. Saikin, and L. J. Sham, Phys. Rev. Lett. 98, 047401 (2007).

${ }^{16}$ X. Xu, Y. Wu, B. Sun, Q. Huang, J. Cheng, D. G. Steel, A. S. Bracker, D. Gammon, C. Emary, and L. J. Sham, Phys. Rev. Lett. 99, 097401 (2007).

${ }^{17}$ V. Loo, L. Lanco, O. Krebs, P. Senellart, and P. Voisin, Phys. Rev. B 83, 033301 (2011).

${ }^{18}$ S. A. Maier, Plasmonics: Fundamentals and Applications (Springer, New York, 2007).

${ }^{19}$ W. Zhang, A. O. Govorov, and G. W. Bryant, Phys. Rev. Lett. 97, 146804 (2006).

${ }^{20}$ R. D. Artuso and G. W. Bryant, Nano Lett. 8, 2106 (2008).

${ }^{21}$ K. T. Shimizu, W. K. Woo, B. R. Fisher, H. J. Eisler, and M. G. Bawendi, Phys. Rev. Lett. 89, 117401 (2002).

${ }^{22}$ A. O. Govorov, G. W. Bryant, W. Zhang, T. Skeini, J. Lee, N. A. Kotov, J. M. Slocik, and R. R. Naik, Nano Lett. 6, 984 (2006).

${ }^{23}$ A. Manjavacas, F. J. García de Abajo, P. Nordlander, Nano Lett. 11, 2318 (2011).

${ }^{24}$ A. Manjavacas, P. Nordlander, F. J. García de Abajo, ACS Nano 2, 1724 (2012).

${ }^{25}$ F. H. L. Koppens, D. E. Chang, F. J. García de Abajo, Nano Lett. 11, 3370 (2011)

${ }^{26}$ V. V. Klimov, M. Ducloy, and V. S. Letokhov, Eur. Phys. J. D 20, 133 (2002).

${ }^{27}$ A. Ridolfo, O. Di Stefano, N. Fina, R. Saija, and S. Savasta, Phys Rev. Lett. 105, 263601 (2010).

${ }^{28}$ D. Brunner, Ph.D. dissertation, Heriot-Watt University (unpublished), http://hdl.handle.net/10399/2350
${ }^{29}$ B. D. Gerardot, D. Brunner, P. A. Dalgarno, P. Öhberg, S. Seidl, M. Kroner, K. Karrai, N. G. Stoltz, P. M. Petroff, and R. J. Warburton, Nature (London) 451, 441 (2008).

${ }^{30}$ M. Kroner, K. M. Weiss, B. Biedermann, S. Seidl, A. W. Holleitner, A. Badolato, P. M. Petroff, P. Öhberg, R. J. Warburton, K. Karrai, Phys Rev. B 78, 075429 (2008).

${ }^{31}$ E. D. Kim, K. Truex, X. Xu, B. Sun, D. G. Steel, A. S. Bracker, D. Gammon, and L. J. Sham, Phys. Rev. Lett. 104, 167401 (2010).

${ }^{32}$ D. Press, T. D. Ladd, B. Zhang, and Y. Yamamoto, Nature (London) 456, 218 (2008).

${ }^{33}$ H. T. Dung, L. Knoll, and D. G. Welsch, Phys Rev. A 66, 063810 (2002).

${ }^{34}$ A. O. Govorov, Phys. Rev. B 82, 155322 (2010).

${ }^{35}$ C. F. Bohren and D. R. Huffman, Absorption and Scattering of Light by Small Particles (Wiley, New York, 1983), Chap. 5.

${ }^{36}$ A. V. Malyshev and V. A. Malyshev, Phys. Rev. B 84, 035314 (2011).

${ }^{37}$ S. M. Sadeghi, Nanotechnology 20, 225401 (2009).

${ }^{38}$ J. Gersten and A. Nitzan, J. Chem. Phys. 75, 1139 (1981).

${ }^{39}$ L. Novotny, Appl. Phys. Lett. 69, 3806 (1996).

${ }^{40}$ F. J. García de Abajo, J. Aizpurúa, Phys. Rev. B 56, 15873 (1997).

${ }^{41}$ L. A. Blanco, F. J. García de Abajo, J. Quant. Spectrosc. Radiat. Transfer 89, 37 (2004).

${ }^{42}$ R. Carminati, J.-J. Greffet, C. Henkel, and J. M. Vigoureux, Opt. Commun. 261, 368 (2006).

${ }^{43}$ A. O. Govorov, J. Lee, and N. A. Kotov, Phys. Rev. B 76, 125308 (2007).

${ }^{44}$ Y. V. Vladimirova, V. V. Klimov, V. M. Pastukhov, and V. N. Zadkov, Phys. Rev. A 85, 053408 (2012).

${ }^{45}$ M. A. Antón, F. Carreño, S. Melle, O. G. Calderón, E. CabreraGranado, J. Cox, and M. R. Singh, Phys. Rev. B 86, 155305 (2012).

${ }^{46}$ P. B. Johnson and R. W. Christy, Phys. Rev. B 6, 4370 (1972).

${ }^{47}$ D. Kleppner, Phys. Rev. Lett. 47, 233 (1981).

${ }^{48}$ P. Anger, P. Bharadwaj, and L. Novotny, Phys. Rev. Lett. 96, 113002 (2006).

${ }^{49}$ M. T. Cheng, S. D. Liu, H. J. Zhou, Z. H. Hao, and Q. Q. Wang, Opt. Lett. 32, 2125 (2007).

${ }^{50}$ G. Lu, T. Zhang, W. Li, L. Hou, J. Liu, and Q. Gong, J. Phys. Chem. 115, 15822 (2011).

${ }^{51}$ Iyyanki V. Jyotsna and G. S. Agarwal, Phys. Rev. A 53, 1690 (1996).

${ }^{52}$ O. G. Calderón, M. A. Antón, and F. Carreño, Eur. Phys. J. D 25, 77 (2003).

${ }^{53}$ A. Urbańczyk, G. J. Hamhuis, and R. Nötzel, Appl. Phys. Lett. 96, 113101 (2010)

${ }^{54}$ M. Pfeiffer, K. Lindfors, C. Wolpert, P. Atkinson, M. Benyoucef, A. Rastelli, O. G. Schmidt, H. Giessen, and M. Lippitz, Nano Lett. 10, 4555 (2010).

${ }^{55}$ M. Pfeiffer, K. Lindfors, P. Atkinson, A. Rastelli, O. G. Schmidt, H. Giessen, and M. Lippitz, Phys. Status Solidi 249, 678 (2012). 